\title{
Analysis of genomic tRNA sets from Bacteria, Archaea, and Eukarya points to anticodon-codon hydrogen bonds as a major determinant of tRNA compositional variations
}

\author{
ILIA TARGANSKI ${ }^{1}$ and VERA CHERKASOVA ${ }^{2,3}$ \\ ${ }^{1}$ BioLing, Inc., Rockville, Maryland 20853, USA \\ ${ }^{2}$ Laboratory of Gene Regulation and Development, National Institute of Child Health and Human Development, National Institutes of Health, \\ Bethesda, Maryland 20892, USA
}

\begin{abstract}
Analysis of 100 complete sets of the cytoplasmic elongator tRNA genes from Bacteria, Archaea, and Eukarya pointed to correspondences between types of anticodon and composition of the rest of the tRNA body. The number of the hydrogen bonds formed between the complementary nucleotides in the anticodon-codon duplex appeared as a major quantitative parameter determining covariations in all three domains of life. Our analysis has supported and advanced the "extended anticodon" concept that is based on the argument that the decoding performance of the anticodon is enhanced by selection of a matching anticodon stem-loop sequence, as reported by Yarus in 1982. In addition to the anticodon stem-loop, we have found covariations between the anticodon nucleotides and the composition of the distant regions of their respective tRNAs that include dihydrouridine (D) and thymidyl (T) stem-loops. The majority of the covariable tRNA positions were found at the regions with the increased dynamic potential-such as stem-loop and stem-stem junctions. The consistent occurrences of the covariations on the multigenomic level suggest that the number and pattern of the hydrogen bonds in the anticodon-codon duplex constitute a major factor in the course of translation that is reflected in the fine-tuning of the tRNA composition and structure.
\end{abstract}

Keywords: tRNA; extended anticodon; hydrogen bonds

\section{INTRODUCTION}

After analyzing the first available near complete set of tRNAs in 1982, Yarus (1982) presented the concept of the extended anticodon, based on the argument that the decoding performance of the triplet anticodon is enhanced by selection of a matching anticodon loop and stem sequence. The recent remarkable progress in the large-scale DNA sequencing of whole genomes has provided an opportunity to study many more full complements of the tRNA genes. In this work we have analyzed 100 complete sets of the cytoplasmic elongator tRNA genes (see Table 1) from Bacteria (45 sets), Archaea (30 sets), and Eukarya (25 sets) in search of the possible

\footnotetext{
${ }^{3}$ Present address: Laboratory of Molecular Growth Regulation, National Institute of Child Health and Human Development, National Institutes of Health, Bethesda, Maryland 20892, USA.

Reprint requests to: Vera Cherkasova, Laboratory of Molecular Growth Regulation, National Institute of Child Health and Human Development, National Institutes of Health, Bethesda, Maryland 20892, USA; e-mail: cherkasv@mail.nih.gov; fax: (301) 496-7823.

Article published online ahead of print. Article and publication date are at http://www.rnajournal.org/cgi/doi/10.1261/rna.896108.
}

occurrence of covariations between the anticodons and the rest of their respective tRNA bodies.

We have viewed nucleotides 36 and 35 of the anticodon as carriers of two main characteristics-base type (purine, $\mathrm{R}$, or pyrimidine, $\mathrm{Y}$ ) and the number of hydrogen bonds (two or three) in a duplex with the complementary nucleotide of the codon. Nucleotide 34 ("wobble base") could not be described in identical terms due to its frequent non-Watson-Crick pairing to its codon counterpart, and, accordingly, its lack of a constant bonding patterns in many cases. Below we refer to the types of anticodon bonding pattern according to the number of hydrogen bonds (hb) formed by nucleotides (nts) 35 and 36; e.g., anticodon 2-2 means " 2 hb-nt 35, 2 hb-nt 36," anticodon 3-3 means " 3 hb-nt 35, 3 hb-nt 36." In all we have four types: 2-2, 3-2, 2-3, 3-3. Accordingly, the strength of interactions of individual nucleotides within an anticodon is expressed in hydrogen bonds: for example, 35-2 or 36-3 means that anticodon nt 35 or 36 forms two and three hydrogen bonds, respectively. The anticodons that form fewer hydrogen bonds during codon-anticodon pairing we call "weaker anticodons" for convenience. The "stronger anticodons" 
TABLE 1. tRNA sequences utilized

List of genomes examined

Abbreviation

GenBank accession number or source

Archaea

Aeropyrum pernix K1

Archaeoglobus fulgidus DSM 4304

Cenarchaeum symbiosum

Ferroplasma acidarmanus

Haloarcula marismortui ATCC 43049

Halobacterium sp. NRC-1

Hyperthermus butylicus DSM 5456

Methanocldococcus jannaschii DSM 2661

Methanococcoides burtonii DSM 6242

Methanococcus maripaludis S2

Methanopyrus kandleri AV19

Methanosarcina acetivorans C2A

Methanosarcina mazei Go 1

Methanospirillum hungatei JF-1

Methanothermobacter thermoautothrophicus delta $\mathrm{H}$

Nanoarchaeum equitans Kin4-M

Nathronomonas pharaonis DSM 2160

Picrophilus torridus DSM 9790

Pyrobaculum aerophilum IM2

Pyrobaculum islandicum DSM 4184

Pyrococcus furiosus DSM 3638

Pyrococcus horikoshii OT3

Staphylothermus marinus $F 1$

Sulfolobus acidocaldarius DSM 639

Sulfolobus solfataricus P2

Sulfolobus tokodaii 7

Thermococcus kodakaraensis KOD1

Thermophilum pandens Hrk 5

Themoplasma volcanium GSS1

Uncultured methanogenic archaeon RC-I

Bacteria

Anaplasma marginale St. Maries

Aquifex aeolicus VF5

Bacillus halodurans C-125

Bacillus subtilis 168

Bacteroides thetaiotaomicron VP1-5482

Bifidobacterium longum NCC2705

Borrelia burgdorferi B31

Buchnera aphidicola APS

Campylobacter jejuni RM1221

Caulobacter crescentus CB15

Chlamidia trachomatis D/UW-3/CX

Chlorobium tepidum TLS

Clostridium tetani E88 Massachusetts

Corynebacterium glutamicum ATCC 13032

Cytophaga hutchinsonii ATCC 33406

Dehalococcoides ethenogenes 195

Deinococcus radiodurans $R 1$

Desulfotalea psychrophila LSv54

Escherichia coli K-12

Geobacillus kaustophilus HTA426

Geobacter sulfurreducens PCA

Gloeobacter violaceus PCC 7421

Helicobacter pylori 199

Lactobacillus plantarum WCFS1

Leptospira interrogans srv. Copenhageni

Fiocruz I, 1-130

Listeria innocua Clip 11262

Ap
$A f$
$C s m$
$\mathrm{Fa}$
$\mathrm{Hm}$
$\mathrm{Hs}$
$\mathrm{Hb}$
$\mathrm{Mj}$
$\mathrm{Mb}$
$\mathrm{Mm}$
$\mathrm{Mk}$
$\mathrm{Ma}$
$\mathrm{Mz}$
$\mathrm{Mh}$
$\mathrm{Mt}$

$\mathrm{Neq}$
$\mathrm{Np}$
$\mathrm{Pt}$
$\mathrm{Pa}$
$\mathrm{Pi}$
$\mathrm{Pf}$
$\mathrm{Ph}$
$\mathrm{Sm}$
$\mathrm{Sa}$
$\mathrm{Ss}$
$\mathrm{St}$
$\mathrm{Tk}$
$\mathrm{Tpn}$
$\mathrm{TV}$
$\mathrm{MrC}$

Tr
MrC
NC_000854

NC_000917

DP_000238

NZ AABC00000000

NC_006396

NC_002607

NC_008818

NC_000909

NC_007955

NC_005791

NC_003551

NC_003552

NC_003901

NC_007796

NC_000916

NC_005213

NC_007426

NC_005877

NC_003364

NC_008701

NC_003413

NC_000961

NC_009033

NC_007181

NC_002754

NC_003106

NC_006624

NC_008698

NC_002578

NC_009464

NC_004842

NC_000918

NC_002570

NC_000964

NC_004663

NC_004307

NC_001318

NC_002528

NC_003912

NC_002696

NC_000117

NC_002932

NC_004557

NC 003450

NC_007298

NC_002936

NC_001263

NC_006138

NC_000913

NC_006510

NC_002939

NC_005125

NC_000921

NC_004567

NC_005824

NC_003212 
TABLE 1. Continued

\begin{tabular}{|c|c|c|}
\hline List of genomes examined & Abbreviation & GenBank accession number or source \\
\hline Mycobacterium leprae $T N$ & & NC_002677 \\
\hline Mycoplasma pulmonis UAB CTIP & & NC_002771 \\
\hline Neisseria meningitides MC58 & & NC_003112 \\
\hline Nitrosomonas europaea ATCC 19718 & & NC_004757 \\
\hline Nostoc sp.PCC 7120 & & NC_003272 \\
\hline Oceanobacillus iheyensis HTF831 & & NC_004193 \\
\hline Prochlorococcus marinus MIT 9312 & & NC_007577 \\
\hline Psychrobacter arcticus $273-4$ & & NC_007204 \\
\hline Rhodopseudomonas palustris BisB5 & & NC_007598 \\
\hline Streptomyces avermitilis MA-4680 & & NC_003155 \\
\hline Synechococcus elongatus PCC 6301 & & NC_006576 \\
\hline Thermoanaerobacter tencongensis MB4 & & NC_003869 \\
\hline Thermobifida fusca $Y X$ & & NC_007333 \\
\hline Thermosynechococcus elongatus BP-1 & & NC_004113 \\
\hline Thermotoga maritima MSB8 & & NC_000853 \\
\hline Thermus thermophilus HB8 & & NC_006461 \\
\hline Treponema pallidum subsp. pallidum Nichols & & NC_000919 \\
\hline Tropheryma whipplei TW08/27 & & NC_004551 \\
\hline Xylella fastidiosa Temecula 1 & & NC_004556 \\
\hline \multicolumn{3}{|l|}{ Eukarya } \\
\hline Anopheles gambiae str. PEST (mosquito) & & NC_004818, NC_009071-72 \\
\hline Aspergillus nidulans & & www.broad.mit.edu/annotation/ \\
\hline Arabidopsis thaliana (land plant) & & www.lowelab.ucsc.edu/GtRNAdb \\
\hline Caenorhabditis elegans (worm) & & www.lowelab.ucsc.edu/GtRNAdb \\
\hline Coprinus cinereus & & www.broad.mit.edu/annotation/ \\
\hline Cryptococcus neoformans JEC 21 & & NC_006670, NC_006679-94 \\
\hline Cryptosporidium parvum lowa type II & & NC_006980-87 \\
\hline Dictyostelium discoideum & & www.genedb.org/genedb/dicty \\
\hline Drosophila melanogaster (fruit-fly) & & www.lowelab.ucsc.edu/GtRNAdb \\
\hline Encephalitozoon cuniculi GB-M1 & & NC_003239-38, NC_003242 \\
\hline Entamoeba histolytica & & www.genedb.org/genedb/ \\
\hline Eremothecium gossypii & & NC_005782-88 \\
\hline Gallus gallus (chicken) & & www.lowelab.ucsc.edu/GtRNAdb \\
\hline Magnaporthe grisei & & www.broad.mit.edu/annotation/ \\
\hline Mus musculus (mouse) & & www.lowelab.ucsc.edu/GtRNAdb \\
\hline Neurospora crassa & & www.broad.mit.edu/annotation/ \\
\hline Pan troglodytes (chimpanzee) & & www.lowelab.ucsc.edu/GtRNAdb \\
\hline Paramecium tetraurelia & & www.cns.fr/paramecie/ \\
\hline Plasmodium falciparum & & www.lowelab.ucsc.edu/GtRNAdb \\
\hline Saccharomyces cerevisiae & & www.lowelab.ucsc.edu/GtRNAdb \\
\hline Schizosaccharomyces pombe & & www.lowelab.ucsc.edu/GtRNAdb \\
\hline Takifugu rubripes (fish) & & www.lowelab.ucsc.edu/GtRNAdb \\
\hline Tetrahymena thermophila SB210 & & NZ_AAGF00000000 \\
\hline Trypanosoma cruzi & & www.genedb.org/genedb/tcruzi/ \\
\hline Ustilago maydis & & www.broad.mit.edu/annotation/ \\
\hline
\end{tabular}

form more hydrogen bonds. The same distinction is applied to the single nucleotide: $35-2$ and 36-2 are "weak" while 35-3 and 36-3 are "strong."

The parameters used for the anticodon nucleotides description were matched manually against every position of the tRNA gene sequence in search of possible covariations. Covariations in the context of our study can be defined as consistent coincidental changes ("correspondences") of the anticodon nucleotides $(35,36$, or both) and any other position in the tRNA. Once found and grouped, the better- expressed ("primary") covariations (mostly with nt 36) were or were not further subgrouped according to weaker expressed ("secondary") correspondences (usually to nt 35).

The patterns of distribution of the "tRNA body" versus anticodon within every examined tDNA set were compared to the other sets within the respective domain of life. Finally, the (sub-) domain-specific patterns were compared to each other. Therefore, the possible instances of nonrandom distribution could be traced on several levels: a single species, a single domain of life, two domains of life, 
and, finally, universal regularities. Mostly the last three types are dealt with in the current article. When possible we attempted to place the results in the context of the available structural data.

Because only the genomic sequences were included in our study, the abundant post-transcriptional modifications of nt 34-known to impact codon binding (for review, see Agris et al. 2007)—were not analyzed. Modification and editing also frequently take place within the tRNA body. As discussed below, a limited incorporation of the mature tRNA sequences in our analysis reveals additional correlations to the anticodon hydrogen bonds. Once available, the data on modification of the complete tRNA sets should further clarify the "extended anticodon" picture.

Since anticodons frequently carry identity signals for the recognition by the cognate tRNA synthetases (Cusack 1997), we had to consider a possibility that "anticodontRNA body" covariations could contribute to the aminoacylation (see Discussion).

The most frequently found type of correspondence is between the number of hydrogen bonds formed by the nucleotides of the anticodon and the composition of the tRNA outside the anticodon. In contrast, the base type ( $\mathrm{R}$ or $\mathrm{Y}$ ) of the anticodon nucleotides does not provide any strong covariations in Bacteria and only one (with nt 37) in Archaea and Eukarya. In the majority of cases it is nt 36 that appears as a primary covariance determinant, thus confirming its definition as a "cardinal nucleotide" (Yarus 1982). Nucleotide 35 regularly appears a secondary determinant. Nucleotide 34 provides overall the lowest degree of covariations, although in Bacteria and nonhyperthermophilic Archaea, its level of correspondence to certain positions of tRNA is significant. The patterns that we consider nonrandom are frequently different between Bacteria on one hand and Archaea and Eukarya on the other hand (see below). The latter two clearly belong together even though there are numerous differences between them.

\section{RESULTS}

\section{Anticodon loop}

Base 37

Nucleotide 37 (always a purine) is located in the immediate vicinity of the nt 36 of the anticodon (AC) (Fig. 1). It has been demonstrated to directly participate in the formation of the codon-anticodon duplex (Murphy et al. 2004; Durant et al. 2005). In Archaea and Eukarya the type of nucleotide in this position ( $\mathrm{G}$ or A) generally corresponds to the base type (R or $\mathrm{Y}$, respectively) of nt $36(P<0.0001)$ (Fig. 2A). In Bacteria, this type of distribution is lacking.

Pair 32-38

Nucleotides 32-38 are demonstrated to form a tertiary non-Watson-Crick (Quigley and Rich 1976; Auffinger and

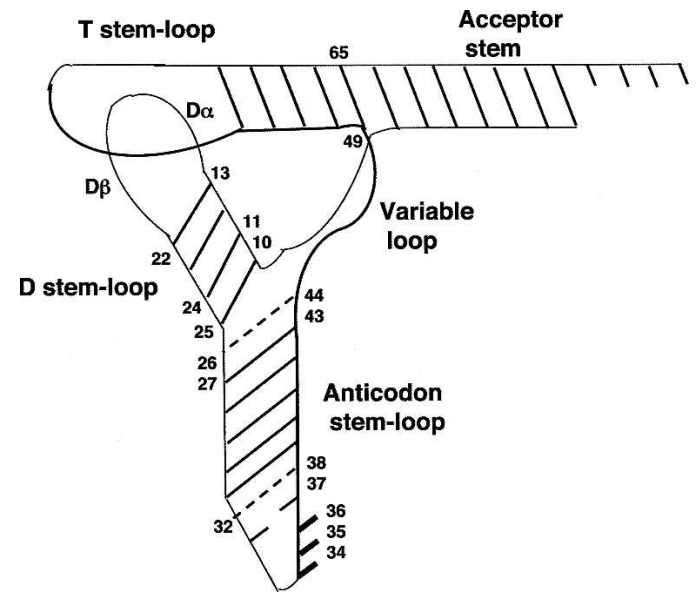

FIGURE 1. The generic class I tRNA in L-shaped two-dimensional representation. Positions of nucleotides discussed in the text are numbered. Dashed lines indicate tertiary interactions.

Westhof 2001) or Watson-Crick (Olejniczak et al. 2005) pair. This is the locus where the covariations with anticodon nucleotides are present in the tRNAs of all three domains of life. The correspondences we found point to hb by nt 36 as a primary determinant of the type of pair 32-38. The weaker (2-2) anticodons have a strong tendency to correspond to C32-A38 $(P<0.0001, P<0.01, P=0.0001$ for Bacteria, Archaea, and Eukarya, respectively; Fig. 2B). Nucleotide 35 appears as a strong secondary determinant primarily in Bacteria (the $32-38$ pair $\mathrm{U}-\mathrm{A}$ is avoided in the case of nt 35-2 $[P<0.01$ and $P<0.0001$ for anticodons $2-2$ and 2-3, respectively]), and to a lesser degree in Archaea and lower Eukaryotes.

The study of a number of mutants of the amber suppressor $\mathrm{Su} 7$ produced the measured suppression efficiency for various combinations of the pair 32-38 as follows: $\mathrm{Cm}-\mathrm{A}>$ Um-A > Um-C > Um- $\Psi$ (Yarus et al. 1986); $\mathrm{m}=$ methylated, $\Psi=$ pseudouridine. This ranking coincides with the frequency of occurrence of the pair 32-38 for the group 36-2: C-A > U-A > U-Y (Fig. 2B; Y = pyrimidine). For the group 36-3 the occurrence of binding-promoting types of 32-38 (C-A, U-A) significantly decreases (Fig. 2B). Thus, the types of the pair 32-38 that stabilize binding dominate anticodon loop (ACL) composition of the tRNAs with weaker binding patterns, while less efficient combinations belong mostly with the stronger anticodons.

The anticodons with U34 display a stronger tendency to have C32-A38 than anticodons with R34 and C34. In Bacteria the difference between tRNAs with G34 and U34 for pair 32-38 is usually such that U32-Y38 (G38) for G34 tends to change into $\mathrm{C} / \mathrm{U} 32-\mathrm{A} 38$ for $\mathrm{U} 34$. In the rare cases when tRNAs with G34 possess U32-A38, tRNAs with the same nt 35-36 and U34 usually have C32-A38. The probability of the 32-38 differentiation matching G/U34 alteration increases with an increase in the number of hb 
A

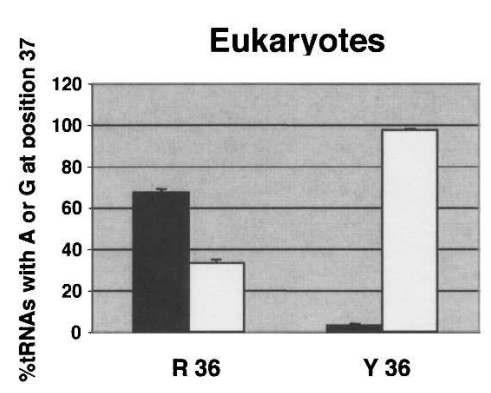

B
Base 37

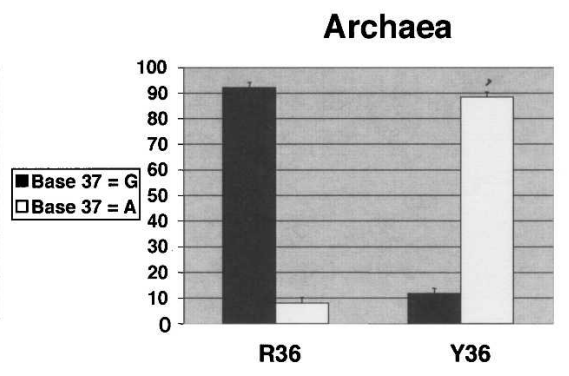

C Base pair $27-43$

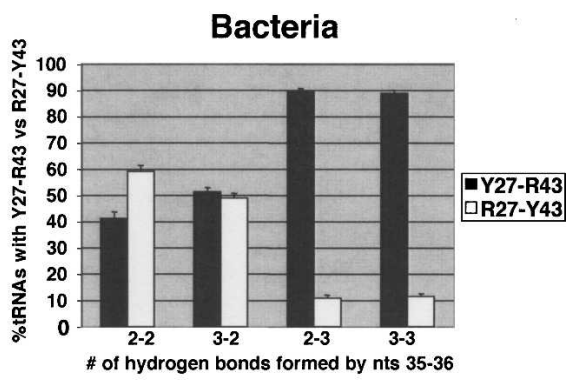

$\%$ tRNAs with certain pair 32-38
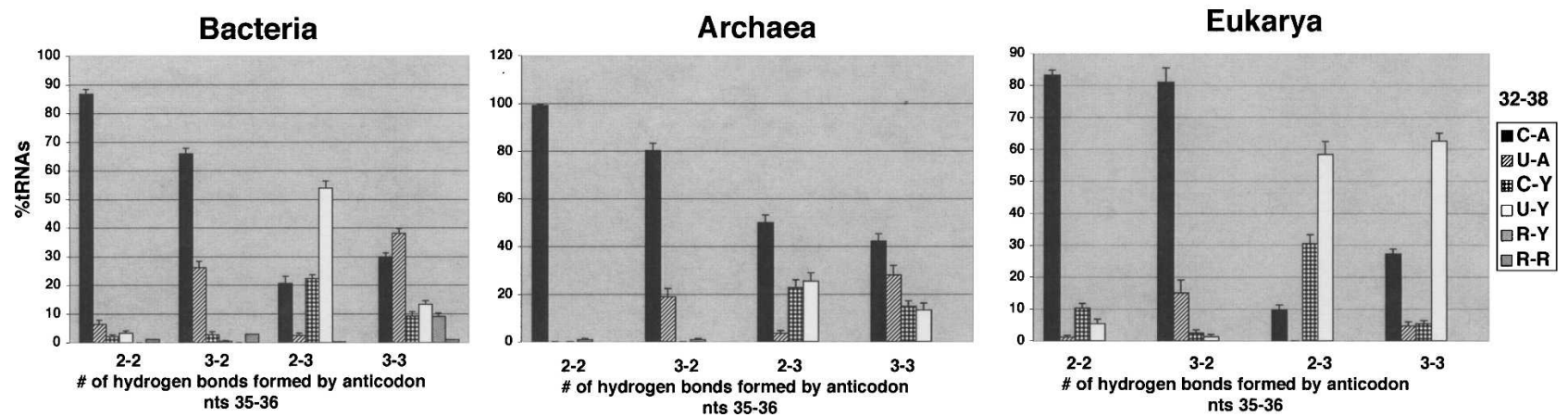

FIGURE 2. Correspondences within the anticodon domain. (A) Correlations between bases 36 and 37 in Archaea and Eukarya. The average percent of tRNAs with R or Y 36 possessing $G$ versus $A$ at position 37 was calculated. For both R36 and Y36 the differences between the obtained and expected values are highly significant $(P<0.0001)$. (B) Correlations between nt 35-36 and pair 32-38. Pairs C-G and U-G are included in the groups $\mathrm{C}-\mathrm{Y}$ and U-Y, respectively, on the basis of the G38 alternation only with the Y38 in the scanned sequences. The statistical significances of the differences between the obtained and the expected values according to the null hypothesis are (1) Bacteria: $P<0.0001$ when $32-38=\mathrm{C}-\mathrm{A}$; $P<0.01$ when 32-38 = U-A; $P<0.0001$ when 32-38 = U-Y,G (anticodons 2-2). For the anticodons other than 2-2, the $P$ values are the following: anticodons 3-2: $0.1(32-38=\mathrm{C}-\mathrm{A}) ; P<0.0001$ (32-38 = U-Y,G); anticodons 2-3: $P<0.0001$ (except for 32-38 = R-Y and R-R); anticodons 3-3: $P<0.001$ (32$38=\mathrm{C}-\mathrm{A}) ; P<0.0001$ (32-38 = U-A and R-Y). (2) Archaea: $P<0.01$ when 32-38 = C-A; $P<0.025$ for 32-38 = U-A; $P<0.1$ for $32-38=\mathrm{U}-\mathrm{Y}, \mathrm{G}$ (anticodons 2-2). For the anticodons other than 2-2, the $P$ values are the following: anticodons 3-2: $P<0.05$ for 32-38 = U-Y,G; anticodons 2-3: $P<$ 0.001 for 32-38 = C-Y,G and U-Y,G; anticodons 3-3: (32-38 = C-A); $P<0.0001$. (3) Eukaryotes: $P<0.0001$ when 32-38= C-A and for 32-38= U-Y,G (anticodons 2-2). For the anticodons other than 2-2, the $P$ values are the following: anticodons 3-2 and 2-3: $P<0.0001$ for 32-38 other than R-Y and R-R; anticodons 3-3: $P<0.025(32-38=\mathrm{C}-\mathrm{A}) ; P<0.0001(32-38=\mathrm{U}-\mathrm{Y}, \mathrm{G})$. The values that do not differ significantly from the expected ones are not included in the legend. $(C)$ Average distribution of the bp $27-43$ in Bacteria. $P<0.0001$ for all the values presented.

formed by nt $35-36(6>5 \geq 4)$ : strong doublets $(6 \mathrm{hb}$, third nucleotide of the codon is degenerate) are well differentiated at $32-38$, weak doublets (5 hb, third nucleotide of the codon is degenerate) are less likely to differ than strong doublets, but more likely than strong triplets (5 hb, nondegenerate third nucleotide of the codon), and weak triplets (4 hb, nondegenerate third nucleotide of codon) are the least likely to differ (Fig. 3). The same tendency is present in Euryarchaeota (Fig. 3), albeit less well pronounced. Therefore, discrimination at position 3238 may take over the function of position 34 for doublets.

The experimental data suggest that C32-A38 tend to induce wobbling at position 34 . Thus, it has been demon- strated that $\mathrm{tRNA}_{\mathrm{UCC}}^{\mathrm{Gly}}$ (i.e., $\mathrm{U} 34+6 \mathrm{hb}$ ) with $\mathrm{C} 32-\mathrm{A} 38$ was unable to discriminate between the glycine codons (Claesson et al. 1995). When U32-A38 was introduced, U34 obtained an ability to discriminate. Surprisingly, C32A38 is prevalent in the tRNAs coding for triplets (Fig. 2B) where discrimination is absolutely required to preserve the fidelity of the coding. However, there is a possibility that C32-A38 may have different effects on wobbling in doublets and triplets. Thus, the U32C mutation increases affinity also to the cognate codon in the A site of the ribosome (Olejniczak and Uhlenbeck 2006) that may be critical for triplets. Additional experimental data are required to clarify this issue. 

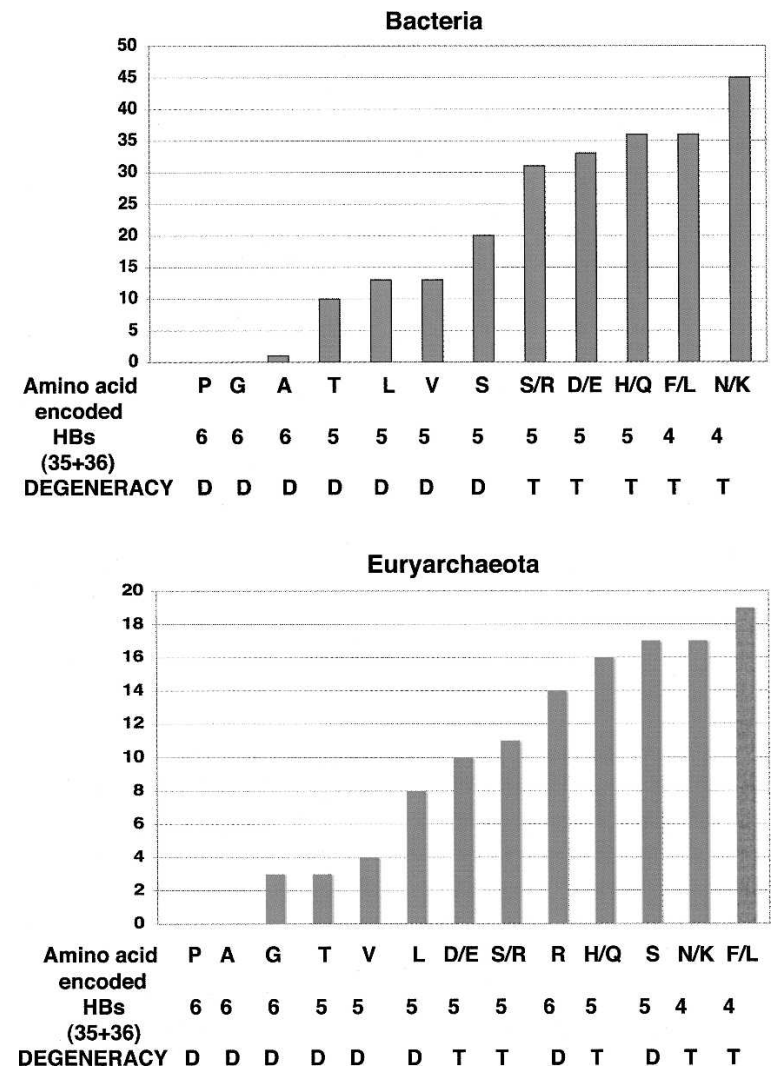

FIGURE 3. Uniformity of pair 32-38 for tRNAs with different nt 34 and identical nt 35-36. Vertical bars indicate the number of cases when pair 32-38 is identical in tRNAs with identical nt 35-36, but different nt 34 ( $\mathrm{G}$ or $\mathrm{U}$ ). D or $\mathrm{T}$ indicates doublet or triplet encoded, respectively. The number of the tRNA pairs used for calculations is the following: Bacteria: Pro-45; Gly-44; Ala-37; Thr-45; Leu-44; Val-35; Ser-44; for each triplet 45 tRNA pairs were compared. Euryarchaeota: 19 pairs of tRNAs were used for calculations. Bacterial tRNA ${ }^{\text {Arg }}$ (anticodon NCG) are not included due to different decoding mode employed (A34 instead of G34 for 38 out of 45 cases).

\section{Anticodon stem}

There is a well-pronounced tendency to have Y27-R43 (Y28-R42 as well, in a number of cases) for tRNAs with nt 36-3 in Bacteria where the frequency of Y27-R43 occurrence is $84.9 \%$ for G36 and $93.5 \%$ for C36 $(P<0.0001)$ (Fig. 2C). No covariations can be seen for tRNAs with nt 36-2: bp Y27-R43 occurs in $45.1 \%$ of A36 and $48.4 \%$ of U36. Thus, if we tried to explain the correspondence strictly in terms of facilitation of the initial binding, we would face an apparent paradox-the weaker, more slippery anticodons should require more of the stabilizing external assistance at this stage, and, accordingly, we would have to expect the presence of regularities (structural restrictions) for this group at first head, while the opposite trend takes place. It is tempting to see this type of distribution as a result of facilitation of the process opposite to the binding, i.e., the release of tRNA from the ribosome. Then the assistance would be required primarily for the potential "stallers," i.e., stronger anticodons, while the more slippery anticodons could be more tolerant to the composition of the distant locus of tRNA. The pair Y27-R43 is in immediate vicinity of the 26-44 hinge point as determined by cryo-EM reconstructions of ribosomes (Valle et al. 2002). tRNA undergoes conformational changes on the ribosome. If tRNA conformation in the $P$ site is the least energetically favorable (Korostelev and Noller 2007), then after peptide bond formation the energy stored within the distorted tRNA molecule may contribute to the driving force during tRNA movement to the E site. Therefore, Y27-R43 pairs may affect energy generated by tRNA in its distorted conformation that may be required for tRNA translocation and release. Another possible explanation of this covariation is the tendency of the tRNA body to compensate the anticodon input to binding that would ultimately result in uniformity of translation of various aa-tRNAs (Dale and Uhlenbeck 2005a,b; Olejniczak et al. 2005). Since there are no data on contribution of Y-R versus R-Y pairs to structural, thermodynamic, or kinetic parameters of tRNA, there is still a possibility that the Y27-R43 pair is a negative determinant that weakens binding of tRNAs forming three hydrogen bonds by nt 36 , thus contributing to uniformity of translation of various aa-tRNAs.

\section{D region}

This region comprises D-stem (DS) base pairs (bp) 10-25, $11-24,12-23$, and $13-22$, and D-loop (DL) of variable length divided in three segments: $\mathrm{D} \alpha$ (nt 14-16, sometimes nt $17,17 \mathrm{ab}$ ), invariable G18G19, and D $\beta$ (nt 20, sometimes $20 \mathrm{ab}$, and nt 21). The DS extensively interacts with the variable loop (VL) to form the tertiary core of the tRNA (Quigley and Rich 1976). The alternative tertiary core design (Biou et al. 1994) of the tRNAs with a long variable arm (class II) led us to restrict our analysis of the DS with the tRNA genes that belong to class I.

In Archaea and Eukarya, in general, class I tRNAs with weaker nt 35-36 bonding patterns possess stronger D stems and vice versa. This type of correspondence is lacking in Bacteria. The most pronounced differentiation was found for the bp 13-22 and 11-24, as discussed next.

\section{Base pair 13-22}

In Archaea and Eukarya (except several Fungi), the anticodons with the weakest bonding pattern (2-2) display the tendency to have stronger bp C13-G22 $(P<0.001)$ (in Crenarchaeota and Lower Eukaryotes this tendency is expanded to both 2-2 and 3-2 anticodons) (Fig. 4B,C). The stronger than 2-2 anticodons are more likely to correspond to the weaker (mismatched) types of 13-22 (Fig. 4). The difference between the eury- and crenarchaeal patterns (Fig. 4, A and B, respectively) illustrates the importance of analyzing the complete tRNA (genes) sets since the opposition of "weak versus strong" appears to be 
$\%$ tRNAs with certain pair 13-22

A

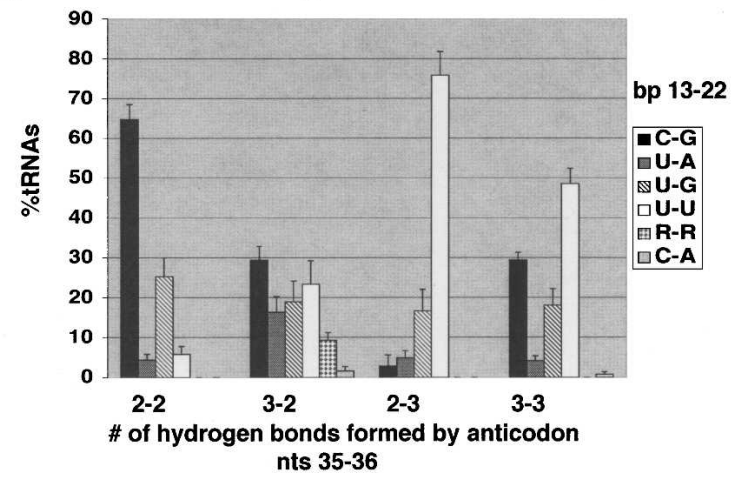

C

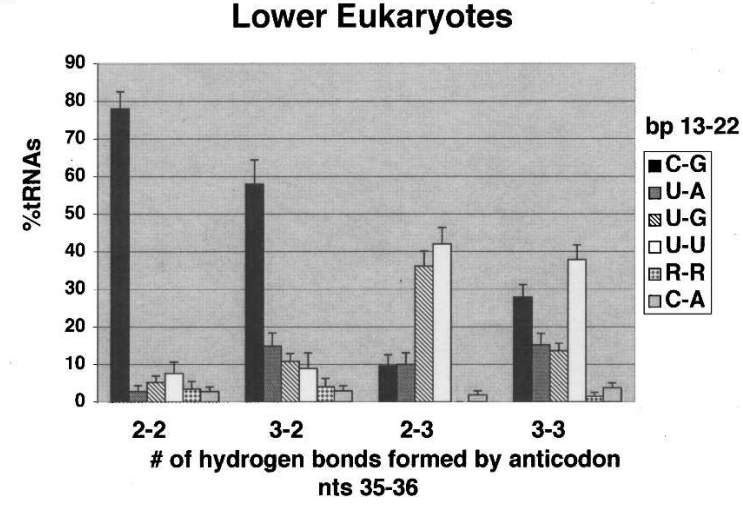

B

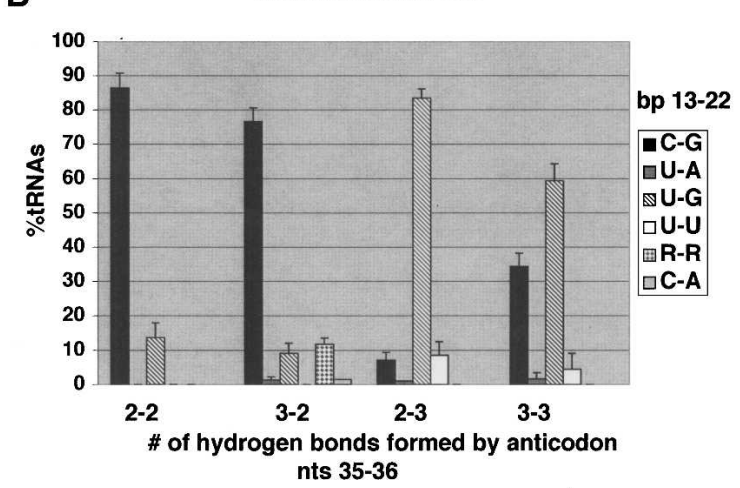

D

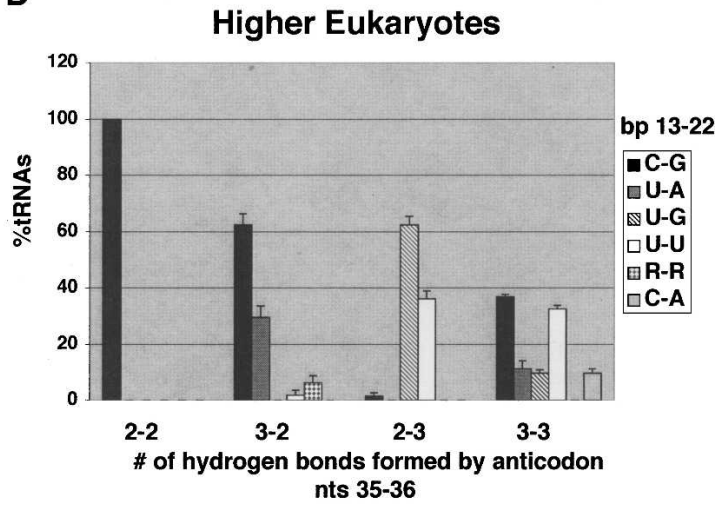

FIGURE 4. Correspondences in the D-region. Distribution patterns of the bp 13-22 in Archaea and Eukarya. The statistical significances of the differences between the obtained and the expected values according to the null hypothesis are $(A)$ Euryarchaeota: for $13-22=\mathrm{C}-\mathrm{G}$ and for $13-$ $22=\mathrm{U}-\mathrm{U}, P<0.001$ (anticodons 2-2 and 2-3). (B) Crenarchaeota: for 13-22 = C-G, $P<0.005$ and $P<0.025$ (when base 36 forms two and three hydrogen bonds, respectively); for 13-22 $=\mathrm{U}-\mathrm{G}, P<0.001$ and $P<0.01$ (when base 36 forms two and three hydrogen bonds). (C) Lower Eukaryotes: for 13-22 $=\mathrm{C}-\mathrm{G}, P<0.001$ and $P<0.005$ (anticodons 2-2 and 2-3, respectively); for 13-22 $=\mathrm{U}-\mathrm{U}, P<0.025$ and $P=0.05$ (when base 36 forms two and three hydrogen bonds, respectively); for 13-22 = U-U, $P<0.001$ when base 36 forms two or three hydrogen bonds. $(D)$ Higher Eukaryotes: for 13-22 = C-G, $P<0.001$ and $P<0.01$ (anticodons 2-2 and 2-3, respectively); for 13-22 = U-A, $P=0.05$ (anticodons 3-2); for 13$22=\mathrm{U}-\mathrm{G}, P<0.0001$ (anticodons 2-3); for 13-22 = U-G or U-U, $P<0.001$ (when base 36 forms two hydrogen bonds) and $P<0.005$ (when base 36 forms three hydrogen bonds). The values that do not differ significantly from the expected are not included in the legend.

relative and may be expressed in two different (groups of) sets at different levels in terms of the absolute scale. It is the opposition between strong C13-G22 and weak U13-U22 that is primarily expressed in Euryarchaeota (Fig. 4A), while in the majority of the crenarchaeons it is the opposition between strong C13-G22 and weaker U13-G22 (Fig. 4B). Thus U13-G22 appears as a medium-strength pair in the euryarchaeal context, but it is the weakest pair 13-22 in the crenarchaeal one. However, the pattern " $36-3$ (strong) $\Rightarrow$ weaker bp 13-22, 36-2 (weak) $\Rightarrow$ stronger bp $13-22$ " is evident in both archaeal subdomains.

\section{Base pair 11-24}

Base pair 11-24 is located in the midsection of the D-stem (Fig. 1). It was experimentally demonstrated to impact decoding (Hirsh 1971; Smith and Yarus 1989; Yarus and
Smith 1995). This bp was suggested to form a tertiary bond to nt 9 (Quigley and Rich 1976; Smith and Yarus 1989) that is possible for C11-G24 and not for U11-A24. It is also possible that this bp facilitates $\mathrm{Mg}^{2+}$ binding when $\mathrm{nt} 11$ is $\mathrm{C}$ and not $\mathrm{U}$ (Gong et al. 2002). Regardless of the exact mechanism of stabilization employed, both models would agree that in Eukarya and Crenarchaeota (Fig. 5A) a more stable bp 11-24 corresponds to a weaker nt 36 and a less stable bp 11-24, in general, to be found in tRNAs with stronger nt $36(P<0.0001)$ (Fig. 5A). More complex patterns have been found in the Euryarchaeota (Fig. 5B), where tRNA genes with G36 frequently (or even predominantly) possess G11-C24 $(P<0.0001)$. Thus for the Euryarchaeota (except for a few methanogenes) the covariations are $\mathrm{A} 36, \mathrm{U} 36 \Rightarrow \mathrm{C} 11-\mathrm{G} 24 ; \mathrm{C} 36 \Rightarrow \mathrm{U} 11-\mathrm{A} 24$, G36 $\Rightarrow$ G/U11-C/A24. Without additional structural data, 
A

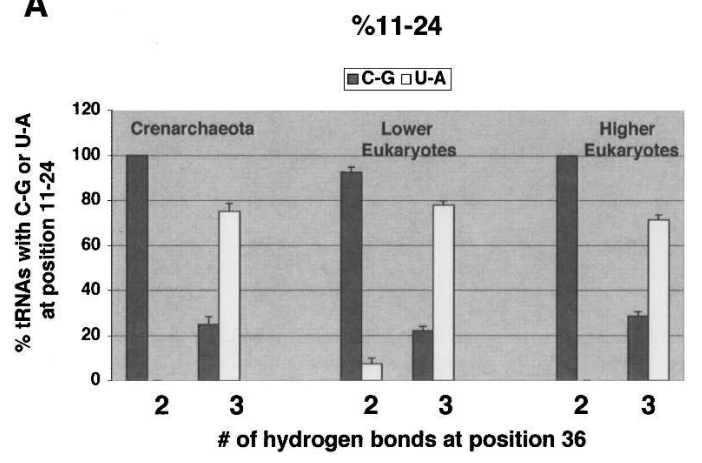

B $\% 11-24$

Euryarchaeota

-C-G $\square$ A-U $\square$ G-C 回U-A

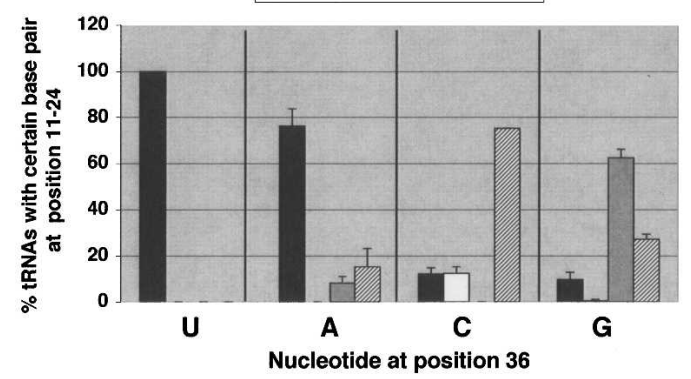

E

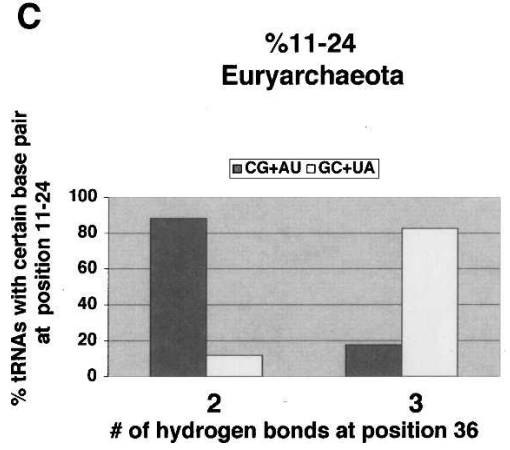

D

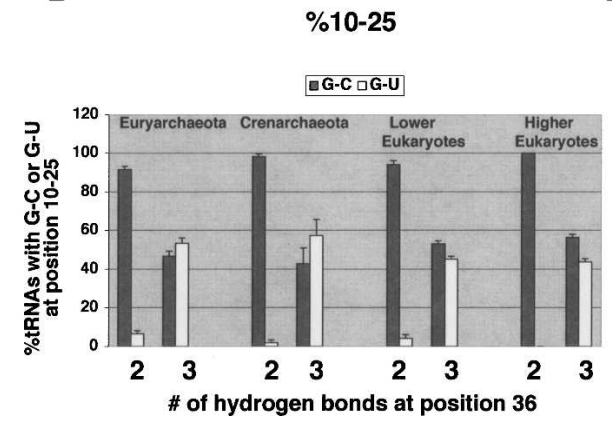

\begin{tabular}{|l|l|l|l|}
\hline $\begin{array}{l}\text { \# of HBs } \\
\text { formed } \\
\text { by bases } \\
35-36\end{array}$ & $\begin{array}{l}\text { Lower } \\
\text { Eukarya } \\
\text { (Fungi) }\end{array}$ & $\begin{array}{l}\text { Lower } \\
\text { Eukarya } \\
\text { (Other) }\end{array}$ & $\begin{array}{l}\text { Higher } \\
\text { Eukarya }\end{array}$ \\
\hline $2-2$ & 6.7 & 6.4 & 6.4 \\
\hline $2-3$ & 6.3 & 6.3 & 6.3 \\
\hline $3-2$ & 5.6 & 5.9 & 5.8 \\
\hline $3-3$ & 5.4 & 5.4 & 5.4 \\
\hline
\end{tabular}

FIGURE 5. Correspondences in the D region (continued). ( $A$ ) Correlations between nt 36 and bp $11-24$ in Crenarchaeota and Eukarya. $P<$ 0.0001 for all the values presented. (B) Correlations between nt 36 and bp 11-24 in Euryarchaeota. All the values significantly differ from the expected $(P<0.0001)$ except when nt $36=$ A (for any bp 11-24) and when nt $36=\mathrm{G}$ (for $11-24=\mathrm{A}-\mathrm{U}$ and $\mathrm{U}-\mathrm{A})$. (C) Distribution of the bp $11-$ 24 in Euryarchaeota based on the combining pairs C-G and A-U (capable to facilitate $\mathrm{Mg}^{2+}$ binding, or form a tertiary bond to nt 9) in one group, and pairs G-C and U-A (unable to accommodate either of two stabilizing interactions) in the other group. $P<0.0001$. ( $D$ ) Correlations between nt 35-36 and bp 10-25. $P<0.0001$ for all the values presented except for Crenarchaeota when $10-25=\mathrm{G}-\mathrm{C}(P<0.01$ and $P<0.025$ for nt 36 forming two and three hydrogen bonds, respectively) and for higher Eukaryotes when 10-25 = G-C $(P<0.005)$. $(E)$ Covariations of the size of the variable segments of D-loop and nt 35-36 in Eukarya. Average D-loop length is given as a sum of the average lengths of D $\alpha$ and D $\beta$ segments of D-loop (in nucleotides). Invariant G18G19 are not included in the calculation. Thus the average full length of the respective D-loops is $N+2$, where $N=$ average number of nucleotides of the variable segments that is presented in this table.

we only can speculate that pairs G11-C24 and U11-A24 may belong together in the sense of being unable either to accommodate $\mathrm{Mg}^{2+}$ ion or to form a tertiary bond to nt 9; i.e., both venues of possible external stabilization of the bp 11-24 are unavailable for the majority of tRNAs with G36 and C36 in Euryarchaeota (Fig. 5C) as well as in Crenarchaeota and Eukarya.

In Bacteria, the bp 11-24 has been shown to affect forward rate constants of GTP hydrolysis and tRNA accommodation steps on the ribosome that are independent of codon-anticodon pairing in the decoding center (Cochella and Green 2005). Therefore, it is not surprising that we have not found correspondences of this bp to anticodon structure in Bacteria. There is a formal possibility that bp 11-24 in Archaea and Eukarya also affects rate constants of GTP hydrolysis and tRNA accommodation as was shown in the bacterial system (Cochella and Green 2005; Selmer et al. 2006), and this indirectly influences codon-anticodon binding, thus, ultimately creating correlations to anticodons. However, because these types of correlations are absent in Bacteria where bp 11-24 is highly standardized (C11-G24 in $\sim 90 \%$ of class I tRNAs), this seems to be unlikely.

\section{Base pair 10-25}

In Archaea and Eukarya the weaker nt 36-2 almost uniformly corresponds to G10-C25 (Archaea 94.0\%, Eukarya 97.2\%; $P<0.0001$ ), unlike the stronger nt 36-3 for which the weaker pair G10-U25 occurs (Archaea 46.1\%, Eukarya $53.7 \%$ ) almost as frequently as G10-C25 (Fig. 5D). While it is technically correct to interpret the difference between G10-C25 and G10-U25 in terms of the sheer strength of the association, the distinctive geometries of these base pairs that result in the conformational difference within the marker sequence signature for the G10 dimethyltransferase (Urbonavicius et al. 2006), and, accordingly, the different types of the G10 modification, seem to be the most likely mechanism underlying the differentiation at this locus.

\section{D-loop}

The covariation of the size of the DL with the strength of the anticodon/codon duplex is well pronounced in Eukarya 
(Fig. 5E). Interestingly, it is rather nt 35 that appears as the "cardinal nucleotide" - unlike all other loci where it is nt 36 that is cardinal. In this case nt 36 often appears as a secondary covariance determinant - the role played by $\mathrm{nt}$ 35 in many other cases of interest.

In Eukarya, the weakest anticodons 2-2 strongly tend to match a $\mathrm{D} \alpha$ region $4-n t$ long $(89.5 \%)$. For the anticodons $3-2,2-3$, and 3-3 the percentages of $\mathrm{D} \alpha-4$ are 18.6, 16.3, and 13.0 , respectively.

There is some evidence suggesting that tRNAs with a 3nt-long, i.e., minimized, $\mathrm{D} \alpha$ region (adjacent to the invariable G18G19 forming a tertiary link with the T-loop in the corner of the tRNA tertiary fold) may possess a more labile tertiary structure in comparison to tRNAs with D $\alpha-4$ (Westhof et al. 1988; Amano and Kawakami 1992). If this is the case, the distribution of the eukaryotic DL size variations lies in line with the variations of D-stems (weak anticodon $\Rightarrow$ strong DS and vice versa) in both Archaea and Eukarya. The eukaryotic tendency toward the minimization of the variable segments of the D-loop $(D \alpha+D \beta)$ proportionally to the increase in the strength of the anticodon hbs (Fig. 5E) would seemingly require a congruent interpretation-which we cannot offer due to the lack of the relevant structural data on the difference between $D \beta-3$ and $D \beta-2$.

Generally, in Eukarya the weaker anticodons appear to have augmented variable segments of the D loop, while the strongest ones tend to have the variable segments minimized in length. Similar correspondence can be seen in some archaeal tRNA sets.

Three types of anticodon-DL correlations have been found in Archaea:

Type 1 (euryarchaeal groups Halobacteria, Methanomicrobia): Similarly to Eukarya, an average $\mathrm{D} \alpha$ length decreases with the increase in the strength of the anticodon hbs (Table 2A).

Type 2 (euryarchaeal groups Methanomicrobia, Methanobacteria, Thermoplasmales, Archaeoglobus; crenarchaeon Thermophilum pendens): tRNAs with weak 2-2 tend to

TABLE 2A. Archaeal D-loop patterns

\begin{tabular}{lcccccc}
\hline \multicolumn{7}{c}{ D $\alpha$ average length (nt) } \\
\hline 35 & 36 & $H s$ & $H m$ & $N p$ & Mh & Mrc \\
\hline 2 & 2 & 4.6 & 4.8 & 4.8 & 4.3 & 4.8 \\
3 & 2 & 4.0 & 4.4 & 4.1 & 4.3 & 4.3 \\
2 & 3 & 4.3 & 4.3 & 4.2 & 3.9 & 3.9 \\
3 & 3 & 3.5 & 3.6 & 3.4 & 3.6 & 3.6 \\
\hline
\end{tabular}

Two columns on the left: hydrogen bonds made by nt 35 and 36 , respectively.

Columns to the right: $\mathrm{D} \alpha$ average length (in nucleotides) given for each individual species. The species abbreviations are listed in Table 1. have $\mathrm{D} \alpha-4$ and/or A at position (18-1), i.e., immediately preceding invariable G18 (nt 16,17, 17a in a conventional numeration depending on the $\mathrm{D} \alpha$ size); tRNAs with strong 3-3 tend to avoid $\mathrm{D} \alpha-4$ and $\mathrm{A}(18-1)$ (Table 2B). Type 3 (Crenarchaeota, euryarchaeon Methanopyrus kandleri): In the sets with the prevalence of $\mathrm{D} \alpha-5$, the compositional biases were found at position (18-1), and/or at position 20, i.e., following invariable G19. The majority of cases should be described as a presence of the outlaying group - with the strongest (3-3) bonding type. This group tends to have $\mathrm{U}(18-1)$ and $\mathrm{A} 20$, while the other groups prefer $\mathrm{A}$ or $\mathrm{C}$ at (18-1) and $\mathrm{C}$ or $\mathrm{U}$ at 20 (Table $2 \mathrm{C}$ ).

The interpretation of the archaeal DL patterns (types 2 and 3 ) is impeded by the scarcity of data on the functional tRNA sequences, particularly in the thermophiles. Now we can only speculate that positions (18-1) and 20, flanking two conserved tertiary pairs in the corner of tRNA, may serve as (negative) markers for the enzymes modifying either 18 or 19 or both. Perhaps, the same role may be played in certain cases by the length of the variable segment $(D \alpha)$, particularly for the selective modification of G15 into archaeosine (see below).

\section{T stem}

This part of tRNA comprises bp 49-65, 50-64, 51-63, 5262 , and 51-61. Base pair 49-65 that has been shown to directly influence the translation (McClain 2006) is the only locus outside the anticodon domain (ACD) where the presence of covariations was found in all three domains of life (Fig. 6A,B). But while the location remains the same, the types of correspondence differ between Bacteria and Archaea-Eukarya. In Bacteria the weaker nt 36 (or nt 3536) corresponds to a stronger (in terms of the hydrogen bonding) bp 49-65 (for the anticodons 2-2, the pair 49-65 forms three hydrogen bonds in $83 \%$ and two or "wobble" in $11.8 \%$ and $5.24 \%$ of cases, which differ from the expected values highly significantly; $P<0.0001 ; P<0.01$ and $P<$ 0.0001 , respectively; Fig. 6A). Conversely, the stronger nt 36 (or nt 35-36) corresponds to a weaker bp 49-65. For the anicodons 3-2, 2-3, and 3-3, the percent of base pairs 49-65 with $3 \mathrm{hb}$ gradually decreases. On the contrary, the percentage of bp 49-65 with $2 \mathrm{hb}$ and "wobble" pairs increases at this position along with the number of hydrogen bonds formed by the respective anticodons, and for the anticodons 3-3 reaches the highest values (Fig. 6A).

In Archaea and Eukarya base 36 appears to be a primary determinant of the distribution: The tRNAs with $2 \mathrm{hb}$ at position 36 mostly possess bp R49-Y65, and the tRNAs with stronger $\mathrm{hb}$ at this anticodon position (nt 36-3) prefer bpY49-R65 (Fig. 6B).

While it is plausible to suggest the optimizing compensation as the mechanism underlying bacterial pattern of distribution of bp 49-65 (see above), there is no clear 
TABLE 2B. Archaeal D-loop patterns

\begin{tabular}{|c|c|c|c|c|c|c|c|c|c|c|c|c|}
\hline \multicolumn{13}{|c|}{$\mathrm{D} \alpha 4$ and $\mathrm{D} \alpha 3$ or 5 with $\mathrm{A}(18-1) /$ versus $\mathrm{D} \alpha 3$ or 5 with (18-1) other than $\mathrm{A}$} \\
\hline 35 & 36 & Mh & Mrc & $M b$ & Mz & Ma & Mt & $\mathrm{Fa}$ & $T v$ & $P t$ & Af & $T p$ \\
\hline 2 & 2 & $5 / 5$ & $7 / 3$ & $7 / 3$ & $6 / 4$ & $7 / 3$ & $6 / 2$ & $8 / 2$ & $10 / 0$ & $8 / 2$ & $8 / 2$ & $8 / 2$ \\
\hline 3 & 2 & $4 / 7$ & $3 / 8$ & $2 / 9$ & $1 / 10$ & $1 / 10$ & $7 / 3$ & $3 / 8$ & $3 / 8$ & $4 / 7$ & $7 / 4$ & $5 / 6$ \\
\hline 2 & 3 & $8 / 4$ & $10 / 2$ & $6 / 6$ & $8 / 4$ & $9 / 4$ & $1 / 9$ & $5 / 7$ & $0 / 12$ & $2 / 10$ & $0 / 12$ & $3 / 9$ \\
\hline 3 & 3 & $3 / 9$ & $2 / 10$ & $3 / 9$ & $3 / 9$ & $3 / 9$ & $0 / 8$ & $1 / 11$ & $2 / 9$ & $2 / 10$ & $0 / 12$ & $3 / 9$ \\
\hline
\end{tabular}

interpretation for the patterns in Archaea and Eukarya. Unlike Bacteria, in Archaea and Eukarya C49 (much more frequently present than U49) is modified to 5-methylcytidine (m5C) (Sprinzl and Vassilenko 2005). The experimental data on the role of the $\mathrm{m} 5 \mathrm{C}$ in the interaction of the anticodon stem-loop analog with the ribosome indicates that this modification increases the thermal stability of the structure, but influences negatively its binding ability (Ashraf et al. 2000). Interestingly, there is a good correlation in Archaea between the growth optimum temperature and the composition of the bp 49-65 (Fig. 6C), which we attempted to interpret in a way similar to the interpretation of the high occurrence of the flexibility-inducing dihydrouridine in the tRNAs from the psychrophiles (Dallugge et al. 1997). A very high percentage of R49-Y65 in a psychrophilic crenarchaeon Cenarchaeum symbiosum suggests that this combination is flexibility inducing, while a (nearly) standard Y49-R65 in the hyperthermophiles points to its possible role in the thermostabilization of the tRNA. Accordingly, the distribution patterns of bp 49-65 in the archaeal and eukaryotic systems indicate that weaker nt 36-2 correspond to a more flexible R49-Y65, while stronger nt 36-3 correspond to a more stable Y49-R65-trends that appear counterintuitive. However, the most straightforward, albeit simplistic, interpretation of the D-loop size variations in the Eukarya and some Archaea (see above) is consistent with them-the longer unpaired segments in the tRNAs with weaker anticodons can be viewed as providers of more diverse local conformational repertoire, while the shorter D-loops that correlate to stronger anticodons are restricted in their dynamics by a sheer lack of size, and hence may be viewed as more rigid.

The archaeal/eukaryotic bp 49-65 pattern found in the $T$ stem formally repeats the bacterial bp 27-43 pattern present in the AC stem (ACS) (see above). There is a formal resemblance between the $\mathrm{T}$ domain and the $\mathrm{AC}$ domain. Both are stem-loop structures comprising a 5-bp-long stem and a 7-nt loop with a characteristic U-turn motif. The bp in question $(49-65,27-43)$ are located at the top of the respective stems (Figs. 1, 7). Both bp are potentially more mobile than regular bp due to the absence of the covalent bond between nt 49 and the adjacent (in the tertiary fold) nt 7 in the case of 49-65, and in the case of 27-43 due to the increased mobility of the unpaired or paired weakly nt

TABLE 2C. Archaeal D-loop patterns

Correlations between types of anticodon and nucleotides at positions (18-1) and 20

\begin{tabular}{|c|c|c|c|c|c|c|c|c|}
\hline \multirow[b]{2}{*}{ Species } & \multicolumn{4}{|c|}{$A+C(18-1) / \cup(18-1)$ for various types of anticodons } & \multicolumn{4}{|c|}{ A20/20 other than A for various types of anticodons } \\
\hline & $2-2$ & $2-3$ & $3-2$ & $3-3$ & $2-2$ & $2-3$ & $3-2$ & $3-3$ \\
\hline Cs & $5 / 5$ & $6 / 6$ & $3 / 8$ & $2^{a} / 10$ & $0 / 10$ & $1 / 11$ & $2 / 9$ & $3 / 9$ \\
\hline Ss & $5 / 5$ & $5 / 7$ & $6 / 5$ & $3 / 9$ & $0 / 10$ & $1 / 11$ & $3 / 8$ & $12 / 0$ \\
\hline St & $5 / 5$ & $6 / 6$ & $7 / 4$ & $3 / 9$ & $0 / 10$ & $0 / 12$ & $4 / 7$ & $12 / 0$ \\
\hline Sa & $6 / 4$ & $8 / 4$ & $9 / 2$ & $4 / 8$ & $0 / 10$ & $0 / 12$ & $4 / 7$ & $12 / 0$ \\
\hline$H b$ & $8 / 2$ & $8 / 4$ & $8 / 3$ & $3 / 9$ & $0 / 10$ & $0 / 12$ & $2 / 9$ & $9 / 3$ \\
\hline$A p$ & $6 / 4$ & $4 / 8$ & $9 / 2$ & $3 / 9$ & $0 / 10$ & $2 / 10$ & $2 / 9$ & $9 / 3$ \\
\hline Sm & $4 / 6$ & $6 / 6$ & $8 / 3$ & $2 / 10$ & $0 / 10$ & $2 / 10$ & $4 / 7$ & $10 / 2$ \\
\hline $\mathrm{Pa}$ & $8 / 2$ & $12 / 0$ & $8 / 3$ & $3 / 9$ & $1 / 9$ & $3 / 9$ & $2 / 9$ & $5 / 7$ \\
\hline$P i$ & $8 / 2$ & $12 / 0$ & $8 / 3$ & $5 / 7$ & $0 / 10$ & $1 / 11$ & $2 / 9$ & $5 / 7$ \\
\hline$T p$ & $8 / 2$ & $9 / 3$ & $9 / 2$ & $3 / 9$ & $1 / 9$ & $1 / 11$ & $6 / 5$ & $3 / 9$ \\
\hline$M k$ & $3 / 5$ & $5 / 3$ & $4 / 4$ & $0 / 8$ & $0 / 8$ & $0 / 8$ & $1 / 7$ & $2 / 6$ \\
\hline
\end{tabular}

The species abbreviations are listed in Table 1. With one exception (see footnote ${ }^{\mathrm{a}}$ ) only A, C, and $\mathrm{U}$ are present at the position (18-1). Consistent opposite trends in distribution are indicated in bold.

ane $\mathrm{G}(18-1)$ included in $\mathrm{A}, \mathrm{C}$ group. 


\section{A $\%$ of tRNAs with certain number of HB} formed by the base pair 49-65

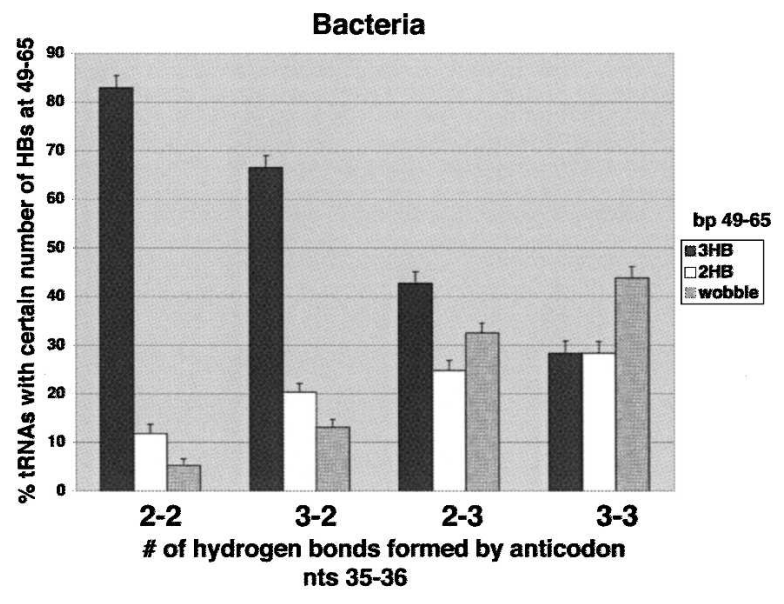

B

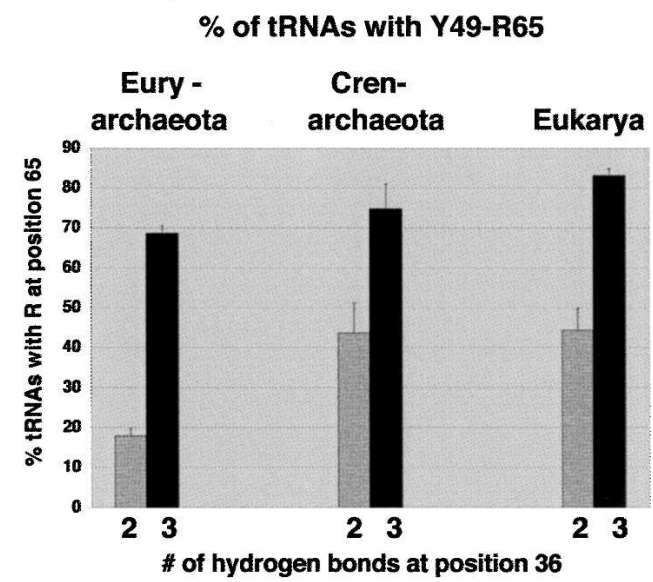

C $\%$ of tRNAs with Y49-R65 Archaea

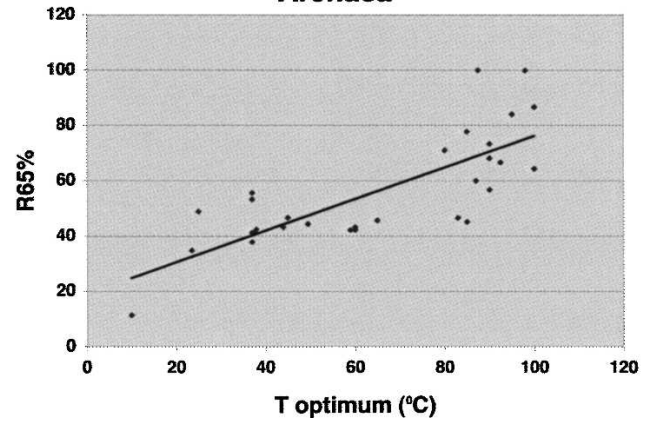

FIGURE 6. Covariations in the aminoacyl arm. (A) Nucleotides 35-36 and bp 49-65 in Bacteria. For the tRNAs with 49-65 forming three, two, or "wobble" pairs, respectively, the $P$ values of the difference of the obtained values from the expected are the following: $P<0.0001, P<0.01, P<$ 0.0001 (anticodons 2-2); $P<0.1$, nonsignificant, and $P<0.025$ (anticodons 3-2); $P<0.1$ (anticodons 2-3); $P<0.0001, P<0.1$, and $P<0.0001$ (anticodons 3-3). (B) Nucleotide 36 and bp 49-65 in Archaea and Eukarya. $P<0.01$ (Euryarchaea); $P<0.01$ (Crenarchaea); $P<0.0001$ (Eukaryotes). (C) Plot of the temperature growth optimum versus composition of the bp 49-65 in Archaea. The distribution fits to the linear trend. Pearson correlation $=0.778 ; P<0.0001$.

26 and 44 atop of these bp. While it has been suggested that the T loop might mimic the ACL (Quigley and Rich 1976), it is unknown if the same structural rhyming can be attributed to the $\mathrm{T}$ and $\mathrm{AC}$ stems. Therefore, it remains unclear whether the two patterns in question may serve the same functional purpose or if the same type of structural correspondence can be utilized for two different purposes.

\section{Aminoacyl stem}

The only possible instance of the covariant distribution in this region is bp 3-70 in Bacteria and Archaea, where the tRNAs with the anticodon type 3-3, i.e., the strongest dinucleotide in terms of hydrogen bonding, display a very strong tendency to have R3-Y70 (96.6\% in Bacteria, 91.3\% in Archaea), which makes them an outlying group since, for the tRNAs with weaker hb patterns, the distribution of this bp is formally random. For the bacterial groups 2-2, 32 , and 2-3 the percentages of bp R3-Y70 are $44.8 \%, 51.0 \%$, and $65.9 \%$, respectively. For the archaeal groups $2-2,3-2$, and $2-3$ the percentages are $64.9 \%, 34.5 \%$, and $50.0 \%$, respectively.

\section{DISCUSSION}

The majority of the tRNA positions that covary with anticodon/codon hydrogen bonding patterns are found at the positions with the increased dynamic potential-such as paired stem-loop $(32-38,13-22)$ and stem-stem (27-43, 10-25, 49-65) junctions that, unlike midstem bp, are less stabilized by the paired near neighbors and thus are more susceptible to a conformational stress.

\section{Two groups of covariations}

The covariant junctional pairs can be divided in two groups according to the type of correspondence. 

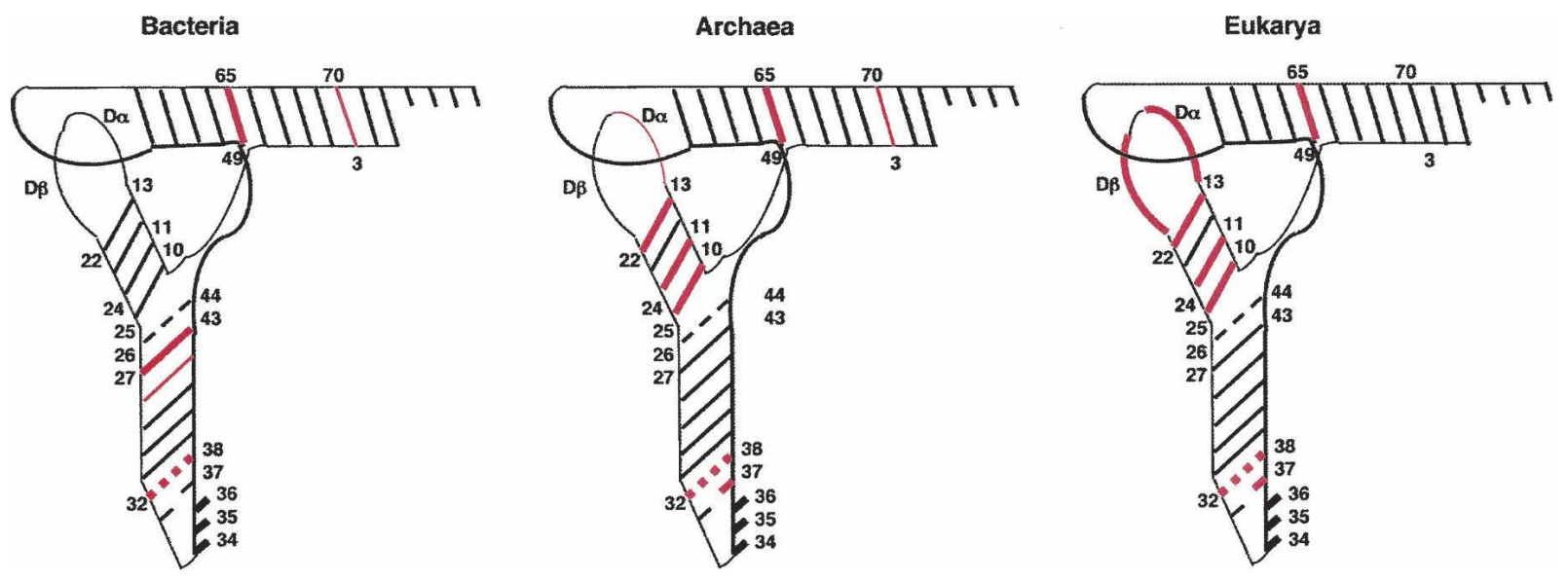

FIGURE 7. Localization of the "hot spots" of the covariations within the tRNA in L-shaped two-dimensional representation in Bacteria, Archaea, and Eukarya. Thick red bars indicate locations of the stronger correlations. Thin red bars mark less pronounced regularities. Red dashed lines indicate tertiary interactions.

The first group comprises bp 10-25, 13-22 in ArchaeaEukarya, and bp 49-65 in Bacteria. In this group the correspondence can be described as anti-correlation between the strength of the hydrogen bonds made by anticodon nucleotide(s) and the strength of the base pair at the distant junction. This type of correspondence may suggest a tolerance to or even a requirement for the disruption of the distant base pair in the tRNAs with stronger nt 36 (nt 3536) at a certain stage of the translation-unlike the tRNAs with weaker nt 36 (nt 35-36), where such a presumed disruption appears to be guarded against. However, there is little experimental data to evaluate the possible role of this anti-correlation in terms of the direct influence of the bp in question on the tRNA function on the ribosome. Recent ribosomal studies have pointed to the tRNA structural distortions and subsequent deformation of the canonic L shape in the course of translation (Frank et al. 2005; Selmer et al. 2006). Our results (group I covariations) may imply that the tRNA body deformability is correlated to the hb strength of the anticodon/codon nucleotides and that the energy required for the transition of tRNA from a high- to a low-energy state is partly stored within the tRNA molecule itself in its deformed state. The energetic potential of each tRNA would come from additive contributions from the distorted tRNA body and anticodon/codon bonds. The result of the covariant contributions would be a roughly uniform energetic potential for each tRNA.

The second group includes pairs 32-38 (all three domains), 27-43 (Bacteria), and 49-65 (Archaea-Eukarya) where the observed patterns are not compensatory. No immediate interpretation can be offered to describe the covariations of these bp in terms of their strength/stability versus weakness/mobility. The experimental evidence points to the fact that in this group the weaker anticodons tend to correspond to the distant pair composition that improves ribosomal binding, while the stronger anticodons display correlation with the occurrence of the bindingimpeding motifs (Yarus et al. 1986; Yarus and Smith 1995; Ashraf et al. 2000). These findings are in a good agreement with the results obtained by Uhlenbeck's group on optimization of aa-tRNA structure (Olejniczak et al. 2005).

In contrast to the junctions, the midstem bp generally do not covary with the hydrogen bonds of the anticodon nucleotides. Out of 13 medial bp in four stems only bp 11-24 (in Archaea and Eukarya) displays the covariation patterns similar to the end residues. Interestingly, the refined crystallographic analysis revealed bases 11 and 24 as highly mobile in the yeast tRNA ${ }^{\text {Asp }}$ (U11-A24), but not in the yeast tRNA ${ }^{\text {Phe }}$ (C11-G24) (Westhof et al. 1988).

\section{Functional significance of covariations}

The presence and type of the tRNA tertiary core (DS, possibly DL) correspondences, as well as the type of covariations of bp 49-65, place Archaea and Eukarya closer to each other than either of them to Bacteria (Fig. 7). The feature common for all three domains is the role of the hydrogen-bonding pattern as a major correspondent parameter. We attribute the consistent occurrence of sequential covariations to their possible function in the course of the translation of the mRNA. Another possible interpretation of the covariations could be their contribution to the aminoacylation, since anticodons frequently carry signals for the recognition by the cognate aminoacyl tRNA synthetases (aaRS). We declined this interpretation for the following reasons: first, our evaluation points to the lack of difference in the expression of covariance between tRNAs amynoacylated by the synthetases belonging to two main classes; second, the tRNA acceptor stems, universally employed by aaRSs for interactions with their cognate 
tRNAs (Perona and Hou 2007), display the lowest degree of covariance among tRNA structural regions; finally, and most importantly, the hydrogen bonding between nt 3536 and aaRS (when it occurs) does not fit into a $2 / 3 \mathrm{hb}$ Watson-Crick pattern that clearly transpires as a major covariant parameter.

In the majority of cases it is nt 36 that appears as a primary covariance determinant, thus confirming its definition as a "cardinal nucleotide" (Yarus 1982). However, the nt 35 regularly appears as a secondary or even primary determinant. For example, the covariation of the size of the D-loop with the hb of the anticodon-codon duplex in Eukarya is correlated primarily to nt 35 (Fig. 5E). This may provide an insight into a mechanism of differentiation between the anticodons 3-2 and 2-3. One possibility is that it is not just the strength of codon-anticodon interactions that matters, but also the topology of the codon-anticodon duplex, which may vary at different stages of translation, thus changing relative contributions of the anticodon nucleotides into the binding mRNA.

\section{Post-transcriptional modifications}

Only the analysis of the complete sets of the fully modified and edited functional tRNAs would provide the complete picture of the anticodon/codon extension. Our look into the available tRNA sequences points to the possibility that in some cases the correspondent local differentiation transpires via the type/presence of the post-transcriptional modification. As mentioned above, there are no apparent patterns of covariations with the anticodon for nt 37 in the bacterial tRNA genes. However, scanning of the available bacterial tRNA sequences (Sprinzl and Vassilenko 2005; http://www.tRNA.un-bayreuth.de) produced a nonrandom distribution of the nt 37(-modified). In Escherichia coli tRNAs with nt 36-3 the modification of nt 37 is minimal, i.e., a single methyl group (1-methyladenosine, 1-methylguanosine) if $\mathrm{nt}$ 36 is $\mathrm{G}$, and minimal (1-methyladenosine, N6-methyladenosine) or lacking altogether if nt 36 is C. In contrast, tRNAs with nt 36-2 contain 2-methylthio-N6-isopentenyl-adenosine as nt 37 when nt 36 is $A$, and even bulkier 9- $\beta-D-$ ribofuranosylpurine-carbamoyl-threonine $(\mathrm{t} 6 \mathrm{~A})$ as $\mathrm{nt} 37$ when nt 36 is U. Both modifications possess long-branched side chains with potentials for participation in the crossstrand stacking in the manner identical or similar to the stabilizing action of t6A37 on the first nucleotide of the codon in the complex of the anticodon domain of the bacterial tRNA ${ }^{\text {Lys }}$ with a complementary codon, as has recently been demonstrated (Murphy et al. 2004; Durant et al. 2005). Thus, although the differentiation is indistinguishable at the tRNA gene level, the patterns of modifications of nt 37 are very different between the tRNAs with nt 36-2 and nt 36-3. Accordingly, the potential of the codon stabilization via cross-strand stacking of the nt 37 onto the codon nt-I is increased for the weak nt 36-2 and diminished or nonexistent for the strong nt 36-3. A similar to bacterial distribution of the modifications of nt 37 also has been found in the available tRNA sequences from the eukaryote Saccharomyces cerevisae (data not shown).

The covariant modifications do not appear to be limited to correspondence to the primary determinant (nt 36), but seemingly may be utilized for the fine-tuning of the structural correspondence to the secondary determinant (nt 35) as well. For example, in the tRNA genes of the higher eukaryotes we find that tRNAs with nt 36-2 uniformly possess C32 (100\%). But a look at the modification pattern of the C32 (Sprinzl and Vassilenko 2005; http://www.tRNA.un-bayreuth.de) reveals an apparent divergence of the group 36-2 into the subgroups 2-2 and $3-2$. While the vast majority $(\sim 88 \%)$ of $\mathrm{C} 32$ in the subgroup 2-2 remain unmodified, in the subgroup 3-2 almost $80 \%$ of tRNAs have 3-methylcytidine as a modification of the base. Remarkably, this modification was found only at this position of tRNA, and only in the eukaryotic tRNAs with the anticodons 3-2.

Interestingly, the presence of the anticodon hb-correlated post-transcriptional modifications in the complex core of the tRNA places bacterium E. coli closer to Archaea and Eukarya, where such patterns are found for the structural elements forming the tRNA tertiary fold. In the available sequences of $E$. coli, the correlations between a type of anticodon hb pattern and the presence of modifications of the G18 and/or a base preceding $48(47,46$, or $47 \mathrm{~N}$ in the conventional numeration depending on the length of the variable loop) are the following:

\begin{tabular}{lc}
\hline Hb 35-36 & Modifications: None/1 or 2 \\
\hline $2-2$ & $1 / 7$ \\
$2-3$ & $4 / 5$ \\
$3-2$ & $7 / 3$ \\
$3-3$ & $6 / 1$ \\
\hline
\end{tabular}

Therefore, the stronger anticodons tend to correspond to unmodified G18 and U47, while the weaker anticodons tend to match at least one of those positions modified, i.e., possess 2'-O-methylguanosine at 18 (modification known to stabilize A-form RNA) and/or 3-(3 amino 3-carboxypropyl) uridine at 47 , i.e., a position potentially influencing a tertiary pair $15-48$. Tertiary pairs G18-Y55 and R15-Y48 are essential for the maintenance of the canonic L-shape of the tRNA tertiary fold, and finding these correlations may well be one of the most intriguing results that we have obtained.

This type of distribution cannot be considered universal in Bacteria-e.g., 2'-O-methylation of G18 is not selective in Thermus thermophilus (Hori et al. 2002) - but the lack of 
data on the modification of the complete tRNA set in the latter leaves a possibility that the same purpose (stabilization of the tertiary fold) can be achieved with different modifications in different bacterial species.

In the halophilic archaeon Haloferax volcanii it is a tertiary pair 15-48 ("Levitt pair") connecting D and variable loops where the modification pattern corresponds to anticodon hb. Some $88.2 \%$ of the tRNAs with $36-2$ have both bases modified (archaeosine that is thought to stabilize tertiary structure [Schimmel and Tamura 2003] at 15; Cm5 at 48), while it is only $22.7 \%$ for the tRNAs with $36-3$. Conversely, it is only a small fraction of the tRNAs with the weak 36-2 that have both bases unmodified (1 out of 17 or $5.9 \%$ ) in contrast to almost a half (10 out of 22 or $45.5 \%$ ) of the tRNAs with the strong 36-3 that are unmodified at both positions.

Therefore, introduction of the mature tRNA sequences in our analysis suggests that the presence of the anticodon hb-correlated idiosyncrasies in the tRNA tertiary core may be a universal mechanism common for all three domains of life.

\section{Concluding remarks}

While the types and levels of correspondences vary between the domains of life as well as within each of them, the very occurrence of the covariations appears to be a trend universally present in all sets of tRNA genes studied. This "invariable covariability" is approximately as universal a feature of tRNA as are, e.g., the design of the main components of its architecture or the presence of the post-transcriptional modifications. Based on the presented data, a strong argument can be made in support of the view that at least one tRNA locus, pair 32-38, possessed a nonrandom, anticodon hb-based pattern of distribution in the tRNAs of the last common ancestor, and if so, the existence of the anticodon-tRNA body covariance as a tRNA composition determinant had preceded the emergence and the subsequent evolution of the extant domains of life.

The covariation between the strength of anticodoncodon interaction and the composition of a distant tRNA locus was first found for the base pair 27-43 in the tRNAs of E. coli (Schultz and Yarus 1994). Our results point to the hydrogen bonds of the codon-anticodon duplex as a major parameter determining certain compositional variations in all structural domains of tRNA as well as in all three domains of life. These new findings further advance and substantiate the concept of the extended anticodon (Yarus 1982). Consequently, it may lead to a further finetuning of the current models of translational machinery, and, particularly, to the elucidation of the role of the number and pattern of the anticodon hydrogen bonds in decoding.

\section{MATERIALS AND METHODS}

\section{tRNA sequences utilized}

Full complements of the tRNA genes from Bacteria (45 sets), Archaea (30 sets), and Eukarya (25 sets) used in this study are listed in the Table 1 together with the source references. The choice of analyzed sets was intended to provide maximal and objective phylogenetic coverage. All major groups within the domains of life with available complete genomes are represented in this study. However, some disproportions-such as a narrow representation of certain groups - could not be avoided due to the limitations imposed by the availability of the genomes.

\section{Analysis of the correspondence between anticodon nucleotides and the loci of the respective tRNAs}

The anticodon nucleotides were matched manually against every position of the respective tRNA gene sequence. The obtained patterns of correspondence of the anticodon parameters and the sequences of the rest of the tDNAs within every examined tDNA set were compared to the other sets within the respective domain (subdomain) of life. When patterns obtained appeared to be significant, the correlations between anticodon structure and other regions of tDNA were calculated as follows: percent of tDNAs with certain anticodon nucleotide and corresponding certain tDNA regions from all isoacceptors were determined within each species initially. Average percents of the tDNAs of certain type and standard deviations were calculated from the percents obtained for every single species. The primary data used for calculations are available upon request.

\section{Statistical analysis}

The statistical significances of the differences between the obtained and the expected values have been calculated using a chi-square test. The chi-square test has been performed against the null hypothesis for every given position in an assumption that distribution at these positions does not differ from the average composition at every particular position of a group of tDNAs tested in every case presented (for each kingdom or subkingdom). Significance of the correlation between the temperature growth optimum and composition of bp 49-65 in Archaea was calculated using the Pearson test.

\section{ACKNOWLEDGMENTS}

We thank Michael Yarus for helpful discussions and comments on the manuscript, Alan Hinnebusch and Thomas Dever for critical reading of the manuscript, and the anonymous reviewers for helpful comments and suggestions.

Received October 30, 2007; accepted February 23, 2008.

\section{REFERENCES}

Agris, P.F., Vendeix, F.A., and Graham, W.D. 2007. tRNA's wobble decoding of the genome: 40 years of modification. J. Mol. Biol. 366: 1-13.

Amano, M. and Kawakami, M. 1992. Assignment of the magnetic resonances of the imino protons and methyl protons of Bombyx 
mori tRNA $\mathrm{Gly}$ Gly and the effect of ion binding on its structure. Eur. J. Biochem. 210: 671-681.

Ashraf, S.S., Guenther, R.H., Ansari, G., Malkiewicz, A., Sochacka, E., and Agris, P.F. 2000. Role of modified nucleosides of yeast tRNA ${ }^{\text {Phe }}$ in ribosomal binding. Cell Biochem. Biophys. 33: 241-252.

Auffinger, P. and Westhof, E. 2001. An extended structural signature for the tRNA anticodon loop. RNA 7: 334-341.

Biou, V., Yaremchuk, A., Tukalo, M., and Cusack, S. 1994. The $2.9 \AA$ crystal structure of T. thermophilus seryl-tRNA synthetase complexed with tRNA ${ }^{\text {Ser }}$. Science 263: 1404-1410.

Claesson, C., Lustig, F., Boren, T., Simonsson, C., Barciszewska, M., and Lagerkvist, U. 1995. Glycine codon discrimination and the nucleotide in position 32 of the anticodon loop. J. Mol. Biol. 247: 191-196.

Cochella, L. and Green, R. 2005. An active role for tRNA in decoding beyond codon:anticodon pairing. Science 308: 1178-1180.

Cusack, S. 1997. Aminoacyl-tRNA synthetases. Curr. Opin. Struct. Biol. 7: 881-889.

Dale, T. and Uhlenbeck, O.C. 2005a. Amino acid specificity in translation. Trends Biochem. Sci. 30: 659-665.

Dale, T. and Uhlenbeck, O.C. 2005b. Binding of misacylated tRNAs to the ribosomal A site. RNA 11: 1610-1615.

Dallugge, J.J., Hamamoto, T., Horikoshi, K., Morita, R.Y., Stetter, K.O., and McCloskey, J.A. 1997. Post-transcriptional modification of tRNA in psychrophilic bacteria. J. Bacteriol. 179: 1918-1923.

Durant, P.C., Bajji, A.C., Sundaram, M., Kumar, R.K., and Davis, D.R. 2005. Structural effects of hypermodified nucleosides in E. coli and human tRNA ${ }^{\text {Lys }}$ anticodon loop: The effect of nucleosides s2U, mcm5U, mcm5s $2 \mathrm{U}, \mathrm{mnm} 5 \mathrm{~s} 2 \mathrm{U}, \mathrm{t} 6 \mathrm{~A}$, and ms2t6A. Biochemistry 44: 8078-8089.

Frank, J., Sengupta, J., Gao, H., Li, W., Valle, M., Zavialov, A., and Ehrenberg, M. 2005. The role of tRNA as a molecular spring in decoding, accommodation, and peptidyl transfer. FEBS Lett. 579: 959-962.

Gong, Q., Guo, Q., Tong, K.L., Zhu, G., Wong, J.T., and Xue, H. 2002. NMR analysis of bovine tRNA ${ }^{\text {Trp }}$ : Conformational dependence of $\mathrm{Mg}^{2+}$ binding. J. Biol. Chem. 277: 20694-20701.

Hirsh, D. 1971. Tryptophan transfer RNA as the UGA suppressor. J. Mol. Biol. 58: 439-458.

Hori, H., Suzuki, T., Sugawara, K., Inoue, Y., Shibata, T., Kuramitsu, S., Yokoyama, S., Oshima, T., and Watanabe, K. 2002. Identification and characterization of tRNA (Gm18) methyltransferase from Thermus thermophilus HB8: Domain structure and conserved amino acid sequence motifs. Genes Cells 7: 259-272.

Korostelev, A. and Noller, H.F. 2007. The ribosome in focus: New structures bring new insights. Trends Biochem. Sci. 32: 434-441.

McClain, W.H. 2006. Surprising contribution to aminoacylation and translation of non-Watson-Crick pairs in tRNA. Proc. Natl. Acad. Sci. 103: 4570-4575.
Murphy 4th, F.V., Ramakrishnan, V., Malkiewicz, A., and Agris, P.F. 2004. The role of modifications in codon discrimination by tRNA Gly GCC. Nat. Struct. Mol. Biol. 11: 1186-1191.

Olejniczak, M. and Uhlenbeck, O.C. 2006. tRNA residues that have coevolved with their anticodon to ensure uniform and accurate codon recognition. Biochimie 88: 943-950.

Olejniczak, M., Dale, T., Fahlman, R.P., and Uhlenbeck, O.C. 2005. Idiosyncratic tuning of tRNAs to achieve uniform ribosome binding. Nat. Struct. Mol. Biol. 12: 788-793.

Perona, J.J. and Hou, Y.-M. 2007. Indirect readout of tRNA for aminoacylation. Biochemistry 46: 10419-10432.

Quigley, G.J. and Rich, A. 1976. Structural domains of transfer RNA molecules. Science 194: 796-806.

Schimmel, P. and Tamura, K. 2003. tRNA structure goes from L to lambda. Cell 113: 276-278.

Schultz, D.W. and Yarus, M. 1994. tRNA structure and ribosomal function. II. Interaction between anticodon helix and other tRNA mutations. J. Mol. Biol. 235: 1395-1405.

Selmer, M., Dunham, C.M., Murphy 4th, F.V., Weixlbaumer, A., Petry, S., Kelley, A.C., Weir, J.R., and Ramakrishnan, V. 2006. Structure of the 70S ribosome complexed with mRNA and tRNA. Science 313: 1935-1942.

Smith, D. and Yarus, M. 1989. Transfer RNA structure and coding specificity II. A D-arm tertiary interaction that restricts coding range. J. Mol. Biol. 206: 503-511.

Sprinzl, M. and Vassilenko, K.S. 2005. Compilation of tRNA sequences and sequences of tRNA genes. Nucleic Acids Res. 33: D139-D140. doi: $10.1093 /$ nar/gki012.

Urbonavicius, J., Armengaud, J., and Crosjean, H. 2006. Identity elements required for enzymatic formation of N2, N2-dimethylguanosine from N2-monomethylated derivative and its possible role in avoiding alternative conformations in archaeal tRNA. J. Mol. Biol. 357: 387-399.

Valle, M., Sengupta, J., Swami, N.K., Grassucci, R.A., Burkhardt, N., Nierhaus, K.H., Agrawal, R.K., and Frank, J. 2002. Cryo-EM reveals an active role for aminoacyl-tRNA in the accommodation process. EMBO J. 21: 3557-3567.

Westhof, E., Dumas, Ph., and Moras, D. 1988. Restrained refinement of two crystalline forms of yeast aspartic acid and phenylalanine transfer RNA crystals. Acta Crystallogr. A 44: 112123.

Yarus, M. 1982. Translational efficiency of transfer RNA's: Uses of an extended anticodon. Science 218: 646-652.

Yarus, M. and Smith, D. 1995. tRNA on the ribosome: A waggle theory. In tRNA: Structure, biosynthesis, and function (eds D. Söll and U. RajBhandary), pp. 443-469. American Society for Microbiology, Washington, DC.

Yarus, M., Cline, S.W., Rafferty, L., Wier, P., and Bradley, D. 1986. The translational efficiency of tRNA is a property of the anticodon arm. J. Biol. Chem. 261: 10496-10505. 

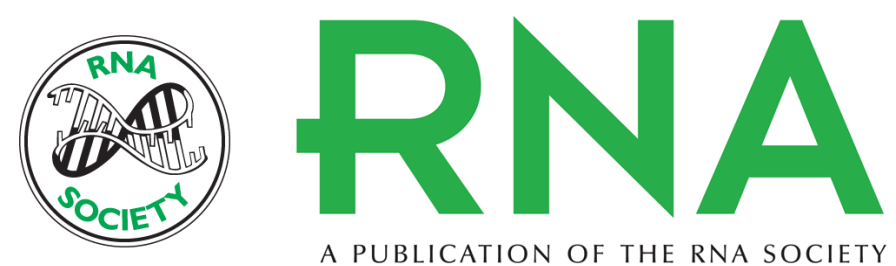

A PUBLICATION OF THE RNA SOCIETY

\section{Analysis of genomic tRNA sets from Bacteria, Archaea, and Eukarya points to anticodon-codon hydrogen bonds as a major determinant of tRNA compositional variations}

Ilia Targanski and Vera Cherkasova

RNA 2008 14: 1095-1109

References This article cites 33 articles, 12 of which can be accessed free at:

http://rnajournal.cshlp.org/content/14/6/1095.full.html\#ref-list-1

License

Email Alerting Receive free email alerts when new articles cite this article - sign up in the box at the

Service top right corner of the article or click here. 